\title{
Online/At-line Measurement, Analysis and Control of Product Titer \& Critical Product Quality Attributes (CQAs) During Process Development
}

\author{
Letha Chemmalil ${ }^{1}$ \\ ${ }^{1}$ Bristol-Myers Squibb Co
}

July 7, 2020

\begin{abstract}
Process analytical technology (PAT) has been defined by the Food and Drug Administration (FDA) as a system for designing, analyzing, and controlling manufacturing through timely measurements to ensure final product quality. Based on quality-bydesign $(\mathrm{QbD})$ principles, real-time or near-real-time data monitoring is essential for timely control of critical quality attributes (CQAs) to keep the process in a state of control. To facilitate next-generation continuous bioprocessing, deployment of PAT tools for real-time monitoring is integral for process understanding and control. Real-time monitoring and control of CQAs is essential to keep the process within the design space and align with the guiding principles of QbD. The contents of this manuscript are pertinent to the online/at-line monitoring of upstream titer and downstream product quality with timely process control. We demonstrated that a UPLC system interfaced with a process sample manager (UPLC-PSM) can be utilized to measure titer and CQAs directly from bioreactors and downstream unit operations, respectively. We established online titer measurements from fed-batch and perfusion-based alternating tangential flow (ATF) bioreactors as well as product quality assessments of downstream operations for real-time peak collection. This integrated, fully automated system for online data monitoring with feedback control is designed to achieve desired product quality.
\end{abstract}

\section{Hosted file}

PAT Manuscript.docx available at https://authorea.com/users/297575/articles/467495-onlineat-line-measurement-analysis-and-control-of-product-titer-critical-product-qualityattributes-cqas-during-process-development 